\title{
LA SOSTENIBILIDAD EN LA GESTIÓN DE LOS RESIDUOS SÓLIDOS URBANOS EN LA CIUDAD DE TEPIC, NAYARIT, MÉXICO. UN ENFOQUE DESDE LA ALIANZA GOBIERNO, SOCIEDAD, ACADEMIA
}

\author{
Sustainability of urban solid waste management in the city of Tepic, Nayarit, Mexico. \\ An approach from a government, society, and academia alliance \\ Graciela BIRRUETA FLORES, Sarah MESSINA FERNÁNDEZ, Víctor H. LUJA, \\ O. NÁJERA-GONZÁLEZ y Claudia E. SALDAÑA DURÁN**
}

Universidad Autónoma de Nayarit, Ciudad de la Cultura Amado Nervo, 63190 Tepic, Nayarit, México

*Autora para correspondencia; cesduran@uan.edu.mx

(Recibido enero 2017; aceptado mayo 2018)

Palabras clave: grupos de interés, actores sociales, manejo de residuos sólidos, gestión urbana sostenible

\section{RESUMEN}

En el presente trabajo se analizó la alianza de los grupos de interés gobierno, sociedad y academia, desde la perspectiva de la gestión de los residuos sólidos en la ciudad de Tepic, Nayarit, México, con miras a la sostenibilidad. Para la evaluación cuantitativa se utilizó el grado de alcance de la alianza expresada en la relación de los grupos de interés conformada por los diferentes actores sociales, con base en una versión adaptada de la metodología de Cohen y Franco. Los resultados indican que a través de la participación de los grupos de interés en las actividades diseñadas, se logró un avance del $31 \%$ en la gestión de residuos sólidos urbanos. Este trabajo demuestra que se puede establecer una gestión integral de los residuos sólidos urbanos con la participación correspondiente de los diferentes actores que conforman los grupos de interés.

Key words: stakeholders, social actors, solid waste management, sustainable urban management

\begin{abstract}
In the present work, an alliance of the interest groups constituted by government, society and academy was analyzed from the perspective of solid waste management in the city of Tepic, Nayarit, Mexico, with the objective of achieving sustainability. For quantitative evaluation, the degree of significance of the alliance, expressed in the relationship of interested groups formed by the different social actors was used, based on an adapted version of the Cohen and Franco methodology. The results indicate that, due to the participation of stakeholders in the designed activities, an advance of $31 \%$ was achieved in the management of urban solid waste. This work demonstrates that an integral management of urban solid wastes can be established with the corresponding participation of the stakeholders that make up the groups of interest.
\end{abstract}




\section{INTRODUCCIÓN}

Los sistemas de gestión de residuos sólidos urbanos (RSU) tienen un comportamiento complejo y multifactorial, debido a la diversidad de materiales que los componen, lo cual se traduce en altos costos ambientales para las ciudades. Tanto las alternativas tecnológicas y económicas como las políticas ambientales han ocasionado cambios en el manejo de los residuos, lo que complica aún más el escenario, desencadenando un nuevo paradigma en el desarrollo urbano sostenible a nivel local, pero con repercusiones a nivel global. La gestión sostenible de RSU requiere un enfoque holístico que considere a las partes involucradas, sus relaciones, actitudes y conocimientos, así como diversos factores vinculados con decisiones complejas, de una manera sensata y lógica. La alianza entre gobierno, sociedad y academia integra a los diferentes actores involucrados en la toma de decisiones sobre la gestión de RSU tanto a nivel individual como colectivo. Esto abarca aspectos sociales, económicos, políticos y ambientales, por lo que es necesario establecer las relaciones entre dichos actores y compararlos. De ahí que la planificación y el desarrollo urbanos demanden una gestión sostenible en las ciudades. Este planteamiento implica la reducción de muchos problemas en el entorno urbano, y subraya la necesidad de protegerlo de la destrucción ecológica ocasionada por concentración de la población en grandes zonas urbanas, escasez de vivienda, pérdida de recursos naturales, contaminación al aire, suelo y agua, entre otros, como consecuencia de un mundo cada vez más urbanizado y con mayor degradación ambiental (Schell y Ulijaszek 1999, Savard et al. 2000, van Herzele y Wiedemann 2003, Wong et al 2006, Kong et al. 2010).

El municipio de Tepic cuenta con 413608 habitantes y una extensión de 2274 km² (INEGI 2015). Predomina la economía del sector terciario. La producción de RSU en la ciudad de Tepic, cabecera del municipio, es un serio problema ambiental, ya que la generación diaria ha aumentado de 300 t en 2003 a $600 t$ en 2011 y $800 t$ en 2015, y se espera que aumente a $950 \mathrm{t}$ en 2020 (DAP 2016). En estudios previos sobre caracterización física de los RSU en la ciudad de Tepic, se demuestra que los residuos orgánicos representan el mayor porcentaje de la composición física, con un $48.74 \%$, mientras que los plásticos representan el $10.9 \%$, el $17.01 \%$ corresponde a materiales no recuperables, el $8.88 \%$ a materiales recuperables, el $13.88 \%$ celulosa sanitaria y EL $0.59 \%$ a residuos peligrosos (Saldaña et al. 2013). Además hay que considerar los residuos de manejo especial (RME), los cuales no son recolectados por la
Dirección de Aseo Público (DAP) sino por empresas locales dedicadas a la recolección en 12 supermercados y nueve centros de servicios (dos cines, seis tiendas departamentales y una bodega de servicio de telecomunicaciones), en los que el mayor porcentaje de la composición física corresponde a residuos orgánicos (37\%), papel y cartón (18\%), plásticos (14\%), sanitarios $(9 \%$ ) y otros residuos (7\%) (Saldaña et al. 2013). En cuanto a los RME en el campus principal de la Universidad Autónoma de Nayarit, la generación diaria se compone principalmente de materia orgánica, con una aportación del $75 \%$, en tanto eu los inorgánicos ocupan el $25 \%$. De estos materiales se pueden recuperar $12 \%$ de papel y cartón, $7.8 \%$ de PET, $1 \%$ de HDPE, $60.70 \%$ de residuos de comida, $1.16 \%$ de aluminio y $3.0 \%$ de vidrio (Saldaña et al. 2012). Estos datos permiten determinar el potencial de reciclaje en la ciudad de Tepic; son significativos y útiles en la toma de decisiones para mejorar las prácticas de manejo adecuado de los residuos.

El objetivo de este trabajo es evaluar ex-ante y ex-post el grado de participación de los grupos de interés gobierno, sociedad y academia — así como sus interrelaciones - con relación a la gestión de los RSU en la ciudad de Tepic, a través de la metodología del grado de alcance de la alianza, y modelar adecuadamente sus interacciones para estructurar estrategias en el proceso de toma de decisiones entre los grupos de interés y sus actores sociales.

\section{LOS GRUPOS DE INTERÉS Y LA GESTIÓN DE LOS RESIDUOS SÓLIDOS}

Se puede analizar a instituciones públicas, reguladores ambientales, grupos ambientalistas y diversos grupos interesados en el entorno natural, para explicar su interacción con el medio ambiente y elaborar planes para garantizar la reducción de problemas ambientales. Por ello, la importancia de los grupos de interés y sus actores sociales en la toma de decisiones tiene efectos positivos sobre la orientación sensible y proactiva en las organizaciones. De acuerdo con la teoría Freeman de los grupos de interés (Freeman et al. 2010), los objetivos de un sistema deberían derivarse equilibrando las demandas conflictivas de los diversos actores del sistema. Por lo tanto, tienen diferentes impactos sobre el sistema, positivos o negativos y el sistema responde a sus intereses.

En el sistema de gestión de los residuos sólidos el principal desafío es desarrollar modelos de apoyo a la toma de decisiones, que consideren las interacciones cooperativas entre las partes interesadas, que son 
cualquier grupo o individuo que puede afectar a los sistemas de RSU o ser afectado por éstos. Se requiere la participación de un gran número de expertos y grupos de interés para la reducción de los residuos, por lo que es importante establecer un vínculo entre todos los grupos de interés para producir un enfoque holístico de la gestión de residuos sólidos (Karmperis et al. 2013, Cole et al. 2014, Aleluia y Ferrao 2016, Yu y Solvang 2016, Moh y Abd 2017)

Los diferentes actores que conforman los grupos de interés involucrados en las operaciones (directores públicos, alcaldes, concejales, miembros del congreso, gerentes ambientales, dueños de negocios, investigadores, agencias reguladoras, etc.) pueden beneficiarse según sus necesidades particulares y aportar una gran cantidad de datos, todo ello con la idea de que las soluciones propuestas sean transparentes y racionales (Shi et al. 2017, Vitorino de Souza et al. 2017).

En el caso de la gestión de recursos naturales, el análisis de los grupos de interés, sus actores y las redes sociales, es un enfoque útil para estudiar sus políticas públicas en la toma de decisiones para la sostenibilidad del medio ambiente (Reed et al. 2009, Lienert et al. 2013, Scanlon et al. 2016, Todd et al. 2017).

El interés, el poder, la actitud y el conocimiento de los grupos de interés conforman una red de interacción o de relaciones que deben examinarse antes de hacer políticas o poner en marcha programas de reciclaje. Por ello es útil conocer a los actores clave o motivar a otros actores (Xu et al. 2016). Este concepto de relación descompone las funciones de los actores sociales para conocer cómo problematizan, se interesan, se involucran y se movilizan para lograr sus objetivos. Las relaciones del gobierno-sociedad se moldean en un espacio a través de una serie de acciones, y dibujan el flujo de la red, por lo que marcan sectores de poder o tensión (Saldaña 2009). Las partes interesadas informales y formales condicionan un enfoque cooperativo, cultivan una estructura institucional flexible y adaptable, elementos que indican que el potencial del manejo de los RSU puede ser sostenible (Steuer et al. 2017).

\section{MATERIALES Y MÉTODOS}

Para determinar las estrategias de organización entre los grupos de interés analizados en este trabajo, gobierno, sociedad y academia, se utilizaron metodologías participativas basadas en la teoría de Freeman (Freeman et al. 2010). Como estrategia del proyecto, los grupos de interés fueron convocados para conformar un equipo de trabajo. Siguiendo las metodologías participativas y educativas como la de los aprendizajes significativos, se diseñaron diferentes actividades para sensibilizar, concientizar y capacitar a los participantes. En el cuadro I se describe cada grupo de interés participante y su función.

En este estudio, la sociedad organizada estuvo representada por los Comités de Acción Ciudadana (CAC) de las colonias Comerciantes, INDECO, Fraccionamiento Jacarandas III Sección y el Campamento Miguel Ángel Paredes, como lo muestra el cuadro II. Para la selección de la muestra se consideraron los siguientes criterios: disponibilidad de participación en el proyecto, experiencia previa en programas de separación de residuos, características sociodemográficas y ubicación en diferentes sectores de la ciudad. La nomenclatura utilizada para identificar a cada grupo de interés y su relación fue: Academia (A), Gobierno (B) y Sociedad (C).

Se aplicó un diseño cuasi experimental al grupo constituido por la alianza gobierno, sociedad y academia para analizar su grado de interacción en torno a la gestión de RSU. Para cuantificar el grado de interacción de la alianza, se llevó a cabo la ponderación de las actividades, asignando un valor de 2 cuando hubiera participación de los tres grupos de interés y un valor de 1 para el caso contrario.

Los indicadores N1, N2, N3 y N4 se definen como las actividades realizadas con la combinación de participación de los grupos de interés y sus actores sociales, $\mathrm{AB}, \mathrm{AC}, \mathrm{BC}$ y $\mathrm{ABC}$, respectivamente, y se denominan unidad de análisis. Enseguida se contabilizan las frecuencias de ocurrencia de cada indicador, y se construye un índice sintético que muestra el grado de interacción de los participantes, el cual toma valores de 0 para cuando no hubo actividades y 4 para el valor más alto de actividades realizadas (Cuadro III).

La ubicación de la unidad de análisis en el indicador permite visualizar la posición relativa de la primera unidad de análisis con relación a la medida de tendencia central del segundo indicador. Los totales se correlacionan de manera directa con el grado de alcance de la alianza. El grado de alcance de la alianza resulta de dividir la sumatoria de los valores de cada unidad de análisis entre la sumatoria del recorrido máximo del indicador (CR), como lo expresan las ecuaciones 1 y 2 .

$$
\begin{aligned}
& \frac{\sum_{i=1}^{i=\mathrm{n}} N i}{C R} \\
& C R=\frac{\text { Valor máximo }+ \text { Valor mínimo }}{2}
\end{aligned}
$$


CUADRO I. GRUPOS DE INTERÉS PARTICIPANTES EN LA GESTIÓN DE LOS RESIDUOS SÓLIDOS URBANOS EN LA CIUDAD DE TEPIC, NAYARIT

\begin{tabular}{lll}
\hline Grupo de Interés & Actor social & Función \\
\hline $\begin{array}{l}\text { Academia } \\
\text { (A) }\end{array}$ & Académicos-investigadores & $\begin{array}{l}\text { Dirigir el proyecto en general y transferir los co- } \\
\text { nocimientos teórico-metodológicos en el ámbito } \\
\text { de los RSU }\end{array}$ \\
\hline $\begin{array}{l}\text { Gobierno municipal } \\
\text { (B) }\end{array}$ & Ayuntamiento del municipio de Tepic & $\begin{array}{l}\text { Fortalecer los procesos de sensibilización y edu- } \\
\text { cación no formal, información de la normatividad } \\
\text { y acompañamiento del proceso de intervención. } \\
\text { Operar la recolección selectiva, la disposición }\end{array}$ \\
& Dirección de Aseo Público & adecuada y el manejo de los RSU \\
\hline $\begin{array}{l}\text { Grupos de la sociedad } \\
\text { organizada (C) }\end{array}$ & Dirección de Ecología & $\begin{array}{l}\text { Promover enlaces con la comunidad, coordinar } \\
\text { la operatividad del manejo de RSU y fomentar la } \\
\text { cultura ambiental en sus colonias. }\end{array}$ \\
& $\begin{array}{l}\text { Comités de acción ciudadana, colonia } \\
\text { Comerciantes }\end{array}$ & ciudadano vinculación de redes de apoyo \\
& Comités de acción ciudadana, colonia & \\
& INDECO & \\
& Comités de acción ciudadana, & \\
& Comités de acción ciudadana, \\
& fraccionamiento Jacarandas & \\
& Activistas ambientales & \\
& & \\
& &
\end{tabular}

CUADRO II. MATRIZ DE ACTIVIDADES DE LAALIANZA ENTRE GOBIERNO, SOCIEDAD Y ACADEMIA EN LA GESTIÓN INTEGRAL DE LOS RESIDUOS SÓLIDOS URBANOS EN LA CIUDAD DE TEPIC, NAYARIT

\begin{tabular}{|c|c|c|}
\hline Actividad & $\begin{array}{l}\text { Participación } \\
\text { (alianza) }\end{array}$ & Ponderación \\
\hline Taller Formación de formadores & A-B-C & 2 \\
\hline Reuniones que se realizaron en la colonia, en el sitio llamado El Galerón & C-A & 1 \\
\hline Se levantaron encuestas sobre diagnóstico en cuatro colonias & $\mathrm{C}-\mathrm{A}$ & 1 \\
\hline Estudio de caracterización de RSU de la colonia Comerciantes & A-B & 1 \\
\hline Reunión de prediseño del plan de acción en la colonia Comerciantes & A-B & 1 \\
\hline $\begin{array}{l}\text { Presentación del plan de acción en la colonia Comerciantes y realización del programa de trabajo } \\
\text { en la colonia }\end{array}$ & A-B-C & 2 \\
\hline $\begin{array}{l}\text { Reunión con las colonias para presentación de resultados de encuestas sobre el diagnostico partici- } \\
\text { pativo }\end{array}$ & A-B-C & 2 \\
\hline Reunión en las escuelas (primaria y preescolar) con el equipo de trabajo & $\mathrm{A}-\mathrm{B}-\mathrm{C}$ & 2 \\
\hline Intervención en las organización institucional educativa Mario Moreno Cantinflas & $\mathrm{B}-\mathrm{C}$ & 1 \\
\hline Intervención en la organización institucional educativa jardín de niños Diana Laura Riojas & $\mathrm{B}-\mathrm{C}$ & 1 \\
\hline Mesa de diálogo Residuos sólidos hacia la sustentabilidad, con participación del equipo de trabajo & A-B-C & 2 \\
\hline Evaluación de actividades/intervención en las colonias con participación del equipo de trabajo & A-B-C & 2 \\
\hline
\end{tabular}

\section{RESULTADOS}

Para analizar el grado de alcance de la alianza de los grupos de interés gobierno, sociedad y academia, se realizó una matriz de las 12 actividades en las que participaron. En el Cuadro I se muestran las combinaciones de relación y participación de los grupos con sus actores y su ponderación de acuerdo con el nivel de interacción. Los valores de cada indicador se muestran en el cuadro II. El grado de alcance de la alianza se obtuvo a partir de la ecuación 1, que resultó de dividir el total del valor de la unidad de análisis, el cual toma un valor de 5, entre la sumatoria del total del máximo del recorrido del indicador, que para este trabajo es igual a 16. Así, el cociente $5 / 16$ da un valor de 0.31 , lo cual significa que el grado de alcance de la alianza es del $31 \%$ (Cuadro IV). 
CUADRO III. INDICADOR DE LA UNIDAD DE ANÁLISIS DE LA ALIANZA ENTRE GOBIERNO, SOCIEDAD Y ACADEMIA EN LA GIRSU EN LA CIUDAD DE TEPIC, NAYARIT

\begin{tabular}{lc}
\hline & Indicador \\
\hline Unidad de análisis & Valores del indicador \\
\hline N1: A-B $=2$ & 1 \\
N2: B-C $=2$ & 1 \\
N3: C-A $=2$ & 1 \\
N4: A-B-C $=6$ & 2 \\
\hline
\end{tabular}

\section{DISCUSIÓN}

El grado de alcance de la alianza gobierno, sociedad y academia hacia la sostenibilidad en la gestión de los RSU en la ciudad de Tepic se determinó en un $31 \%$, evaluado mediante la metodología de Cohen y Franco (2006). Esta metodología, que se utiliza para la evaluación cuantitativa de los proyectos sociales, muestra cómo estudiar la acción pública, lo que implica observar principalmente el objeto de análisis. A través de la relación entre los grupos de interés, sus actores y las redes entre ellos, se puede alcanzar una gestión con una mezcla de soluciones integradas que aborden los complejos problemas sociales de los RSU con características de sostenibilidad, así como una cultura de reducción en el consumo y conciencia social hacia la sostenibilidad (Saldaña et al. 2013); en consecuencia, se pueden lograr avances para la solución integral del problema ambiental generado por el inadecuado manejo de los residuos. La evaluación de este trabajo permitió establecer un vínculo entre las partes interesadas para producir un enfoque sostenible en la gestión de residuos.
Determinar el interés, el poder, la actitud y el conocimiento de los diferentes actores sociales de los grupos de interés, podría servir para implementar políticas o poner en marcha programas de reciclaje, así como incorporar otros grupos para mejorar la situación compleja de la gestión de residuos. Un vínculo concreto, como una alianza entre los actores principales puede impulsar la gestión adecuada de los RSU. La gestión eficaz supone la consideración de una serie de criterios económicos, ambientales, técnicos y sociales críticos, al tiempo que requiere la cooperación conjunta de todas las partes interesadas, junto con la voluntad política y la eficacia (Achillas et al. 2013).

\section{CONCLUSIONES}

El enfoque desde la alianza de gobierno, sociedad y academia para la sostenibilidad de la gestión de RSU en la ciudad de Tepic, Nayarit, México, representa un modelo que puede ser mejorado con la ejecución de más acciones que fomenten la participación de los actores. La tendencia hacia la cooperación entre los grupos de interés estudiados en este trabajo se cuantificó con el grado de alcance de la alianza, que resultó con un valor del $31 \%$. Este valor es significativo debido a que es la primera vez que se cuantifica el grado de cooperación entre los actores sociales clave en la gestión de los RSU en la ciudad de Tepic, y este logro se atribuye a la intervención de la academia. Este resultado sugiere que es preciso dirigir esfuerzos de investigación y acción en este tema, para fortalecer los vínculos de cooperación y participación entre las partes involucradas, y establecer políticas orientadas a la gestión integral de los RSU, que consideren los diversos factores que influyen en la toma de decisiones.

CUADRO IV. INDICADOR DE LA UNIDAD DE ANÁLISIS DE LAALIANZA ENTRE GOBIERNO, SOCIEDAD Y ACADEMIA EN LA GIRSU EN LA CIUDAD DE TEPIC, NAYARIT

\begin{tabular}{|c|c|c|c|c|c|}
\hline \multirow{3}{*}{ Indicador } & \multicolumn{5}{|c|}{ Valores } \\
\hline & \multicolumn{2}{|c|}{ Recorrido del indicador } & \multirow{2}{*}{$\begin{array}{l}\text { Valor central } \\
\text { del indicador } \\
\text { (mediana) } \\
\text { (3) }\end{array}$} & \multirow{2}{*}{$\begin{array}{l}\text { Obtenido por la } \\
\text { unidad de } \\
\text { análisis } \\
\text { (4) }\end{array}$} & \multirow{2}{*}{$\begin{array}{c}\text { Grado de logro de la alianza } \\
\text { obtenido de la unidad de análisis } \\
\text { Máximo de recorrido del } \\
\text { indicador }\end{array}$} \\
\hline & $\begin{array}{l}\text { Máximo } \\
\text { (1) }\end{array}$ & $\begin{array}{l}\text { Mínimo } \\
\text { (2) }\end{array}$ & & & \\
\hline $\mathrm{N} 1: \mathrm{A}-\mathrm{B}=2$ & 4 & 0 & 2 & 1 & \\
\hline $\mathrm{N} 2: \mathrm{B}-\mathrm{C}=2$ & 4 & 0 & 2 & 1 & \\
\hline N3: $\mathrm{C}-\mathrm{A}=2$ & 4 & 0 & 2 & 1 & \\
\hline $\mathrm{N} 4: \mathrm{A}-\mathrm{B}-\mathrm{C}=6$ & 4 & 0 & 2 & 2 & \\
\hline Total & 16 & 0 & 8 & 5 & $\begin{array}{c}0.31 \\
31 \%\end{array}$ \\
\hline
\end{tabular}


Finalmente, mediante el presente trabajo se logró vincular con éxito a los grupos de interés y sus actores sociales, con un alto nivel de aceptación, en torno a la gestión de los residuos sólidos urbanos, un tópico que merece ser estudiado mediante un enfoque holístico con mayor profundidad en trabajos futuros.

\section{AGRADECIMIENTOS}

Este trabajo de investigación fue financiado por el Consejo Nacional de Ciencia y Tecnología (CO$\mathrm{NACyT}$ ) dentro del proyecto de investigación aprobado como pertinente en la convocatoria Proyectos de Investigación Científica a Problemas Nacionales 2014, número de solicitud 247699.

\section{REFERENCIAS}

Achillas C., Moussiopoulos N., Karagiannidis A., Banias G. y Perkoulidis G. (2013). The use of multi-criteria decision analysis to tackle waste management problems: A literature review. Waste Manage. Res. 31(2), $115-129$

DOI: $10.1177 / 0734242 X 12470203$

Aleluia J. y Ferrao P. (2016). Characterization of urban waste management practices in developing Asian countries: A new analytical framework based on waste characteristics and urban dimension. Waste Manage. $58,415-429$.

DOI: 10.1016/j.wasman.2016.05.008

Cohen E. y Franco R. (2006). Evaluación de proyectos sociales. 7a ed. Siglo XXI Editores, México, 318 pp.

Cole C., Osmani M., Quddus M., Wheatley A. y Kay K. 2014. Towards a zero waste strategy for an English local authority. Resour. Conserv. Recy. 89, 64-75. DOI: 10.1016/j.resconrec.2014.05.005

Christine C., Osmani M., Quddus M., Wheatley A. (2014). Towards a zero waste strategy for an English local authority. Resour. Conserv. Recy. 89, 64.75.

DOI: 10.1016/j.resconrec.2014.05.005

DAP (2016). Informe Dirección de Aseo Público. H. XL Ayuntamiento de Tepic. Tepic, Nayarit.

Freeman R.E., Harrison J.S., Wicks A.C., Parmar B.L. y de Colle S., Eds. (2010). Stakeholder theory. The state of the art. Cambridge University Press, Cambridge, Inglaterra, $343 \mathrm{pp}$.

INEGI (2015). II Conteo de Población y Vivienda 2015. Instituto Nacional de Estadística y Geografía [en línea]. http://www.inegi.org.mx/est/contenidos/proyectos/ ccpv/cpv2005/
Karmperis A.C., Aravossis K., Tatsiopoulos I.P. y Sotirchos A. (2013). Decision support models for solid waste management: Review and game-theoretic approaches. Waste Manage. 33 (5), 1290.1301.

DOI: 10.1016/j.wasman.2013.01.017

Kong F., Yin H., Nakagoshi N. y Zong Y. (2010). Urban green space network development for biodiversity conservation: Identification based on graph theory and gravity modeling. Landscape Urban Plan. 95(12), 16-27.

DOI: 10.1016/j.landurbplan.2009.11.001

Lienert J., Schnetzer F. e Ingold K. (2013). Stakeholder analysis combined with social network analysis provides fine-grained insights into water infrastructure planning processes. J. Environ. Manage. 125, 134-48. DOI: 10.1016/j.jenvman.2013.03.052

Moh Y.C. y Abd Manaf L. (2017). Solid waste management transformation and future challenges of source separation and recycling practice in Malaysia. Resour. Conserv. Recy. 116, 1-14.

DOI: 10.1016/j.resconrec.2016.09.012

Reed M.S., Graves A., Dandy N., Posthumus H., Hubacek K., Morris J., Prell C., Quinn C.H. y Stringer L.C. (2009). Who's in and why? A typology of stakeholder analysis methods for natural resource management. J. Environ. Manage. 90 (5), 1933-1949.

DOI: 10.1016/j.jenvman.2009.01.001.

Saldaña Durán C.E. (2009). Políticas públicas y sociedad. el caso de la gestión de los residuos sólidos urbanos en la ciudad de Tepic, Nayarit. Universidad de Guadalajara [en línea]. http://act.cuaad.udg.mx/es/alumnosadmin/tesis-concluidas\#S

Saldaña Durán C.E., Hernández R.I. y Messina F.S. (2012). Gestión integral de los RSU en la Universidad Autónoma de Nayarit. Memorias del $5^{\circ}$ Encuentro Nacional de Expertos en Residuos Sólidos. Xalapa,Veracruz, 24-26 de octubre [CD-ROM].

Saldaña Durán C.E., Hernández R.I., Messina F.S., Pérez Pimienta J.A. (2013). Caracterización física de los residuos sólidos urbanos y el valor agregado de los materiales recuperables en el vertedero El Iztete, de Tepic, Nayarit, México. Rev. Int. Contam. Ambie. 29 (3), 25-32.

Saldaña Durán C.E., Hernández R.I., Ibarra R.D. (2013). Clasificación de residuos de manejo especial de supermercados y centros de servicios en la ciudad de Tepic, Nayarit. $6^{\circ}$ Encuentro Nacional de Expertos en Residuos Sólidos. Nuevo Vallarta, Nayarit, 11-13 de septiembre, pp. 182-187.

Savard J.P.L., Clergeau P. y Mennechez G. (2000). Biodiversity concepts and urban ecosystems. Landscape Urban Plan. 48 (3-4), 131-42.

DOI: $10.1016 / \mathrm{S} 0169-2046(00) 00037-2$ 
Scanlon T., Uguru O.P., Jafry T., Chinsinga B., Mvula P., Chunga J., Zimba L.M., Mwape M., Nyundo L., Mwiinga B. y Chungu K. (2016). The role of social actors in water access in Sub-Saharan Africa: Evidence from Malawi and Zambia. Water Resour. Rural Dev. 8, 25-36. DOI: 10.1016/j.wrr.2016.08.001

Schell L.M. y Ulijaszek S.J. (1999). Urbanism, urbanization, health and human biology: An introduction. En: Urbanism, health and human biology in industrialised countries (Schell L.M. y Ulijaszek S.J, Eds.). Cambridge University Press, Reino Unido, 330 pp.

DOI: 10.1080/11287462.2014.897070

Shi H., Liu H.C., Li P. y Xu X.G. (2017). An integrated decision making approach for assessing healthcare waste treatment technologies from a multiple stakeholder. Waste Manage. 59, 508-517.

DOI: 10.1016/j.wasman.2016.11.016

Steuer B., Ramusch R., Part F. y Salhofer S. (2017). Analysis of the value chain and network structure of informal waste recycling in Beijing, China. Resour. Conserv. Recy. 117, 137-50.

DOI: 10.1016/j.resconrec.2016.11.007

Todd L., Leask A., Ensor J. (2017). Understanding primary stakeholders' multiple roles in hallmark event tourism management. Tourism Manage. 59, 494-509.

DOI: 10.1016/j.tourman.2016.09.010
Van Herzele A. y Wiedemann T. (2003). A monitoring tool for the provision of accessible and attractive urban green spaces. Landscape Urban Plan. 63 (2), 109-26. DOI: 10.1016/S0169-2046(02)00192-5

Vitorino de Souza Melaré A., Montenegro González S., Faceli K. y Casadei V. 2017. Technologies and decision support systems to aid solid-waste management: A systematic review. Waste Manage. 59, 567-584. DOI: 10.1016/j.wasman.2016.10.045

Wong S.W., Tang B.S. y Horen B.V. (2006). Strategic urban management in China: A case study of Guangzhou development district. Habitat Int. 30 (3), 645-667. DOI: 10.1016/j.habitatint.2005.04.001

Xu W., Zhou C., Cao A. y Luo M. (2016). Understanding the mechanism of food waste management by using stakeholder analysis and social network model: An industrial ecology perspective. Ecol. Model. 337, 63-72. DOI: 10.1016/j.ecolmodel.2016.06.006

Yu H. y Solvang W.D. (2016). An improved multi-objective programming with augmented $\varepsilon$-constraint method for hazardous waste location-routing problems. Int. J. Env. Res. Pub. He. 13 (6), 548.

DOI: 10.3390/ijerph13060548 